\title{
Testicular Interstitial Cell Tumor with Disseminated Cutaneous Metastasis in a Dog
}

\author{
Harlan Hallamys de Lima Nascimento', Camila Tochetto', Ricardo Barbosa Lucena² \& Glaucia Denise Kommers ${ }^{3}$
}

\begin{abstract}
Background: Although testicular tumors commonly develop in the genital tract of dogs, only few studies have reported about metastatic testicular tumors in dogs to date. Therefore, the present study aimed to describe the gross, histopathological, and immunohistochemical findings in a dog with metastatic interstitial cell tumor.

Case: A 6-year-old male Beagle underwent clinical examination due to enlargement of the left testicle and nodules in the skin of the left inguinal region and of the scrotum. The left testicle was cryptorchid (inguinal), and the right testicle was intrascrotal. Both testicles and affected skin fragments were surgically removed. The right testicle was normal in size and did not exhibit gross changes. However, the left testicular parenchyma had been completely replaced by a firm, grayish-to-white neoplastic mass with an irregular surface. The inguinal and scrotal skin fragments were thickened by the neoplastic masses. Histologically, the left testicular parenchyma was completely obliterated by a neoplastic mass consisting of round-to-oval and polygonal cells. The neoplastic cells generally had abundant eosinophilic cytoplasm with indistinct cell limits. The nuclei were round and small, with aggregated chromatin. However, some cells exhibited high degrees of atypia and pleomorphism and large nuclei with granular chromatin. Mitotic figures were observed at high frequencies of up to 11 per high-power field (400x). The mass in the left testicle was diagnosed as malignant interstitial cell tumor. The sections of the inguinal skin and scrotum presented with neoplastic cells similar to those observed in the left testicle, which is consistent with the metastasis of the malignant testicular interstitial cell tumor. On the basis of necropsy, multiple cutaneous and subcutaneous nodules were randomly distributed throughout the body, primarily in the prepuce, head, and limbs. Histological evaluations of the skin nodules, internal iliac lymph nodes, and abdominal skeletal muscles also revealed metastases of the testicular interstitial cell tumor.

Discussion: The histopathological findings associated with the immunohistochemical results observed in the present case helped in establishing the diagnosis of malignant interstitial cell tumor. Regarding the histological evaluations, although most neoplastic cells exhibited appearances that are characteristic of Leydig cells, several features indicative of malignancy were also observed. In dogs, malignant interstitial cell tumors are rare, and multiple organ metastases have been described. Among the immunohistochemical markers that were examined (calretinin, vimentin, cytokeratin, placental alkaline phosphatase, and octamer-binding transcription factor 3/4), only vimentin staining produced a positive result. In one study, the antibody against vimentin stained both normal Sertoli and Leydig cells and tumors arising from these cells, but not seminomas, which is an important differential diagnosis in the case described in this study. The negativity for placental alkaline phosphatase also allowed to rule out seminoma in the differential diagnosis of the primary tumor. The skin sections (distant metastases) were negative for $\mathrm{T}$ and $\mathrm{B}$ lymphocytes and macrophages, thereby ruling out possible differential diagnoses, such as lymphomas or histiocytic tumors, among the cutaneous neoplasms. Therefore, this case report described a malignant testicular interstitial cell tumor with an uncommon disseminated pattern of metastases to the skin in a dog.
\end{abstract}

Keywords: testicular neoplasia, malignant interstitial cell tumor, diseases of dogs. 


\section{INTRODUCTION}

According to the latest World Health Organization classification of tumors in domestic animals, primary tumors of the testicles are classified into three types: sex-cord stromal tumors, which include both Sertoli cell tumors (SCTs) and interstitial (Leydig) cell tumors (ICTs); germ cell tumors, which include seminomas, teratomas, embryonal carcinomas, and yolk sac carcinomas; and mixed tumors [9].

ICT is a mesenchymal neoplasm originating from the interstitial Leydig cells located between the seminiferous tubules [1,5]. Most of these tumors are benign; however, cases of malignant forms, although extremely rare, were recorded [2,19]. Comparing to ICTs, the veterinary literature most commonly associates seminomas and Sertoli cell tumours with malignant behavior (metastases), as well as with clinical syndromes and cryptorchidism [7,12]. ICTs are unusually associated with these findings. However, in few cases involving dogs, multiple metastases in the skin and skeletal musculature were the main findings observed that were indicative of malignancy $[2,19]$.

The use of ancillary tests for histopathological examination, such as the immunohistochemical (IHC) technique, may contribute to the definitive diagnosis of malignant ICTs. Therefore, the present study aimed to describe the gross, histopathological, and IHC findings in a dog with metastatic ICT.

\section{CASE}

A 6-year-old male Beagle underwent clinical examination due to enlargement of the left testicle and nodules in the skin of the left inguinal region and scrotum over a course of approximately 1 month, as observed by the owner. The left testicle was cryptorchid (inguinal), and the right testicle was intrascrotal. Both testicles and affected skin fragments were surgically removed and sent to the Laboratório de Patologia Veterinária of the Universidade Federal de Santa Maria.

The right testicle was normal in size and did not exhibit gross changes. However, the left testicular parenchyma had been completely replaced by a firm, grayish-to-white neoplastic mass with an irregular surface (Figure 1). The affected skin fragments were thickened by the neoplastic masses, which appeared similar to those of the mass observed in the left testicle. Histologically, the left testicular parenchyma was completely obliterated by a neoplastic mass, consisting of round-to-oval and polygonal cells. The neoplastic cells generally had abundant eosinophilic cytoplasm, sometimes vacuolated, with moderately distinct cell limits (Figure 2). The nuclei were round, peripheral, and small, with aggregated chromatin; however, some cells exhibited high degrees of atypia and pleomorphism and large nuclei with granular chromatin. Mitotic figures were observed at high frequencies of up to 11 per high-power field (400x). Multiple areas of necrosis were also observed. The mass in the left testicle was diagnosed as malignant ICT. Sections of the affected skin presented with neoplastic cells similar to those observed in the left testicle, which were arranged in sheets that infiltrated the superficial and deep dermis. The histological findings were consistent with metastases of testicular ICT. The dog was submitted to euthanasia approximately 1 month after ICT diagnosis due to its extremely poor clinical condition.

On the basis of necropsy, multiple cutaneous and subcutaneous nodules (Figures $3 \& 4$ ) randomly distributed throughout the body, primarily in the prepuce, head, and limbs, were found. These nodules were firm and well delimited, ranging from 0.8 to $2.0 \mathrm{~cm}$ in diameter, and were homogeneously white at the cut surface, and some nodules were ulcerated. The left internal iliac lymph node had been replaced by a $5.0 \times 6.0 \times 2.5 \mathrm{~cm}$ white mass. The right internal iliac lymph node was firm and slightly enlarged, and its cut surface was characterized by brown areas that were interspersed with white areas. The muscles of the abdominal wall (left inguinal region) were transmurally invaded by a neoplastic white mass. The abdominal skeletal muscles were invaded by nodules from the prepuce skin. Histological evaluations of the skin nodules (Figure 5), internal iliac lymph nodes, and abdominal skeletal muscles revealed metastases of the testicular ICT, as previously identified in the left testicle. Immunohistochemistry was performed using calretinin $^{1}$, vimentin ${ }^{1}$, cytokeratin ${ }^{1}$, placental alkaline phosphatase $^{2}$ (PLAP), and octamer-binding transcription factor $(\mathrm{OCT}-3 / 4)^{3}$ in the sections of the primary tumor and of metastasis in the skin. Positive immunostaining was observed only for vimentin (Figure 6). In addition, IHC for CD3 ${ }^{1}$ (T lymphocyte), CD79 ${ }^{1}$ (B lymphocyte), and MAC- $387^{1}$ (histiocytic-myelocitic marker) was performed only in the skin sections to rule out other cutaneous round cell tumors. Toluidin blue staining was also performed to rule out mast cell tumor. 


\section{DISCUSSION}

The histopathological findings associated with the IHC results observed in the present case were helpful in establishing the diagnosis of malignant ICT. Regarding the histological evaluations, although most neoplastic cells exhibited appearances that are characteristic of Leydig cells, several features indicative of malignancy were also observed. In dogs, malignant ICTs are rare, and multiple organ metastases have been described $[2,3,10,19]$. Similar to the findings of Laufer \& Sulman [10], the dog in the present report was cryptorchid. Moreover, the dog's left testicle was contained in the inguinal region. Cryptorchid testes are most commonly associated with the development of seminomas and SCTs [7]. However, epidemiological studies have reported the occurrence of ICTs in dogs with cryptorchidism $[6,11]$.

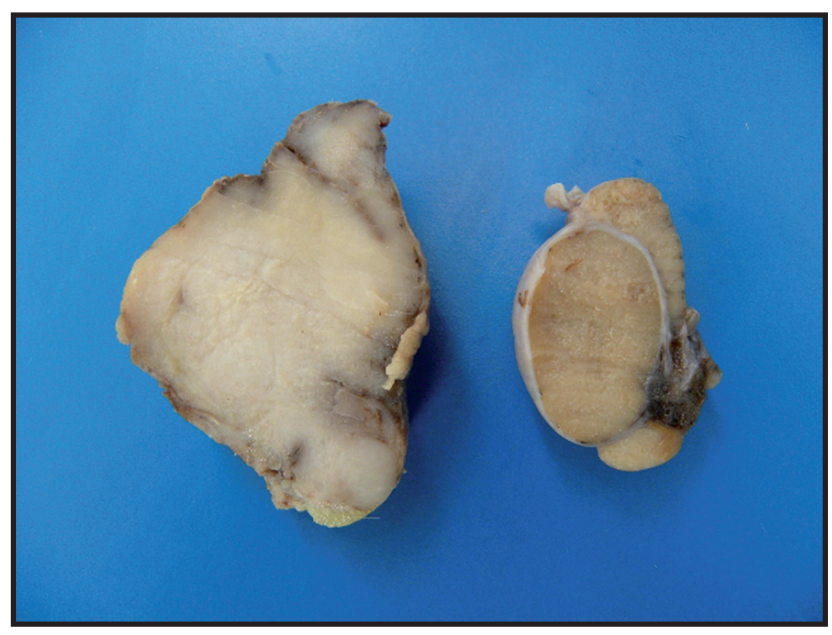

Figure 1. The left testicular parenchyma was completely replaced by a firm, grayish-to-white neoplastic mass (interstitial cell tumor). The right testicle did not exhibit any gross changes.

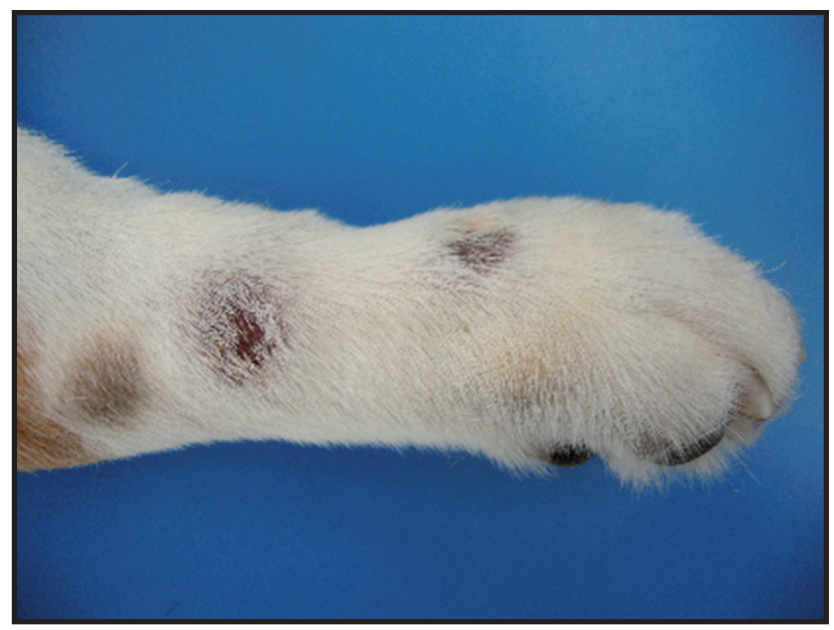

Figure 3. Cutaneous metastasis of the testicular interstitial cell tumor. Two nodules were observed in the thoracic limb, and the largest tumor was ulcerated.
Approximately $10 \%-20 \%$ of men present with ICT metastasis [16], and the metastatic varieties are described in adults and elderly individuals aged 29-82 years. Invasive vascular and adnexal testicular structures as well as necrosis, nuclear atypia, and high mitotic index are microscopic findings indicative of malignancy in ICTs in humans $[8,16]$. The actual differences between benign and malignant ICTs are not well documented in the veterinary literature [3]. In extremely rare cases of malignant ICTs described in dogs, metastases were the main criterion indicative of malignancy $[2,10,19]$.

Malignant ICT in a dog that metastasized to the skeletal musculature of the left pelvic limb has been described [19]. Similar findings were observed in the present case in which invasion of the abdominal wall musculature in the left inguinal region was observed. Re-

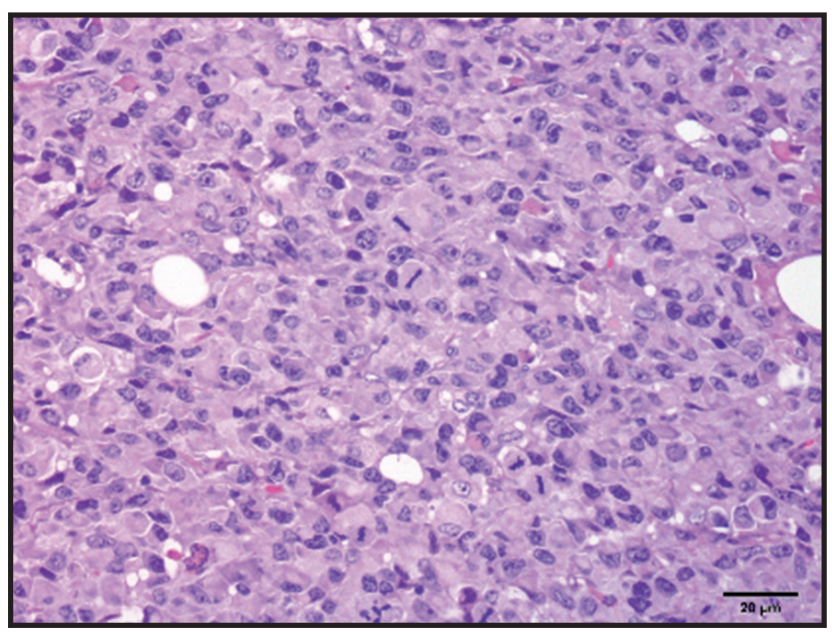

Figure 2. Histology of the testicular interstitial cell tumor. The neoplastic cells were round-to-oval or polygonal, with abundant eosinophilic cytoplasm. Some cells exhibited high degrees of atypia and pleomorphism and had large nuclei and granular chromatin. Mitotic figures were frequently observed [H\&E; Bar $=20 \mu \mathrm{m}$ ].

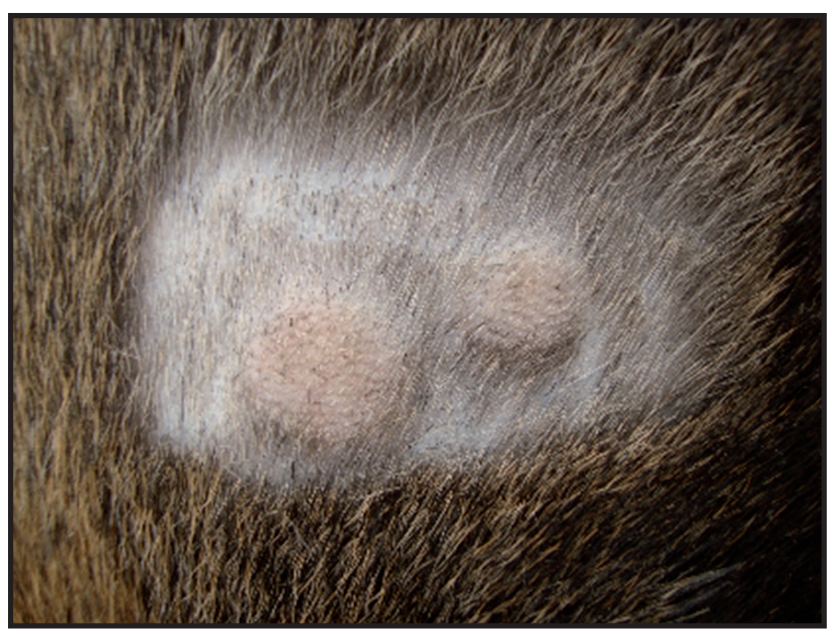

Figure 4. Cutaneous metastasis of the testicular interstitial cell tumor. Two salient nodules on the skin of the lumbar region were observed. 


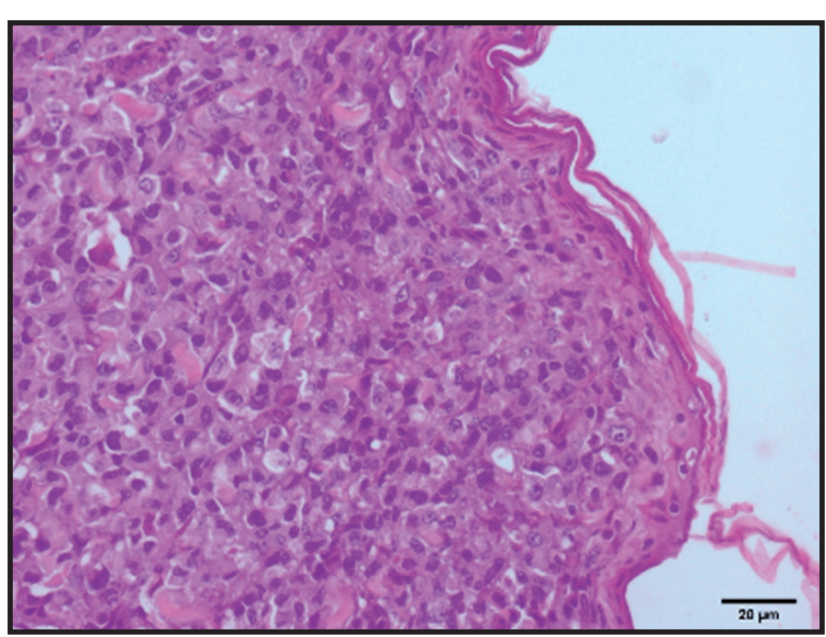

Figure 5. Histology of the cutaneous metastasis of the testicular interstitial cell tumor. Neoplastic cells were observed within collagen fibers [H\&E; Bar $=20 \mu \mathrm{m}]$.

cently, malignant ICT with metastasis in the skin of the dorsum and in the interscapular region was observed in a $\operatorname{dog}$ [2]. In the present report, cutaneous metastases were also observed on the face, thoracic limbs, and foreskin. Cutaneous metastases of malignant testicular tumors are most commonly observed in seminomas $[17,18]$.

In this case report, metastasis to the regional lymph nodes was observed. Metastasis to the internal iliac lymph nodes and the involvement of skeletal muscles adjacent to the region of the primary tumor can be explained by lymphatic spreading and direct tumor extension, respectively. Cutaneous metastases arising from the regions adjacent to the primary tumor site are indicative of lymphatic spreading. However, multiple metastases to various distant skin sites cannot be explained by this mechanism [4]. In the present case, the exact mechanism by which spreading occurred in the skin cannot be determined because of the tumor cells were not identified in the blood vessels of the examined skin sections.

Among the examined IHC markers (calretinin, vimentin, cytokeratin, PLAP, and OCT-3/4), only vimentin staining produced a positive result. In one study, the antibody against vimentin stained both normal Sertoli and Leydig cells and tumors arising from these cells, but not seminomas [14], which is an important differential diagnosis in the case described in the present study. Recently, calretinin (along with inhibin-alpha) was utilized to confirm the Leydig cells originating from a malignant metastatic ICT, with weak to moderate immunostaining [2]. However, in another study, in normal canine testis, calretinin expression was restricted to Leydig and Sertoli cells of the testis, and in tumor tissues, calretinin expression was detected in all tumors that were investigated

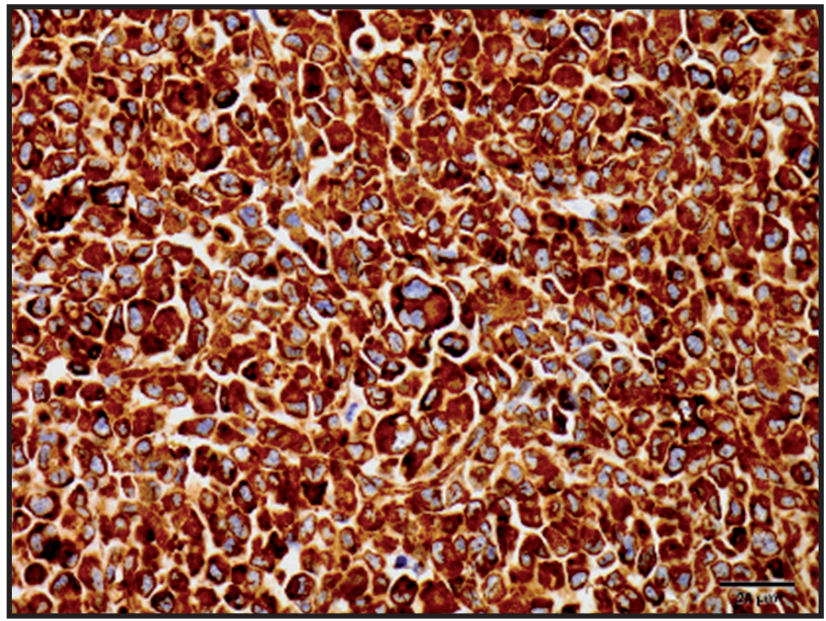

Figure 6. Neoplastic cells were strongly immunolabelled to vimentin. Streptavidin-biotin-peroxidase method [Bar $=20 \mu \mathrm{m}]$.

(ICTs, seminoma, and SCTs) [15], indicating the apparent low specificity of this marker for testicular tumors.

Some IHC panels have been indicated for the diagnoses of testicular tumors in dogs [13]. However, to date, there are no consistent specific IHC markers for canine ICTs [13], indicating that further studies must be conducted in this field. It is important to emphasize that the histomorphology strongly indicated that the tumor cells had interstitial cell characteristics in the present case [12].

The negative immunoreactivity for PLAP allowed to rule out seminoma in the differential diagnosis of the primary tumor (corroborating with the result for vimentin, as previously discussed). The skin sections (distant metastases) were negative for $\mathrm{T}$ and B lymphocytes and macrophages, thereby ruling out possible differential diagnoses, such as lymphomas or histiocytic tumors, among the cutaneous neoplasms. In this regard, the cellular morphology and negativity in toluidine blue staining also helped in ruling out mast cell tumor. Therefore, this case report described a malignant testicular ICT with an uncommon disseminated pattern of metastases to the skin in a dog.

\section{MANUFACTURERS}

${ }^{1}$ DakoCytomation. Carpinteria, CA, USA.
${ }^{2}$ NeoMarkers. Fremont, CA, USA.
${ }^{3}$ Santa Cruz Biotechnology. Dallas, TX, USA.

Acknowledgements. The authors would like to thank the Coordenação de Aperfeiçoamento de Pessoal de Nível Superior (CAPES) for the financial support and Enago (www.enago. com) for the English language review.

Declaration of interest. The authors report no conflicts of interest. The authors alone are responsible for the content and writing of the paper. 


\section{REFERENCES}

1 Al-Agha O.M. \& Axiotis C.A. 2007. An in-depth look at leydig cell tumor of the testis. Archives of Pathology \& Laboratory Medicine. 131(2): 311-317.

2 Canadas A., Romão P. \& Gartner F. 2016. Multiple cutaneous metastasis of a malignant leydig cell tumour in a dog. Journal of Comparative Pathology. 155(2-3): 181-184.

3 Dow C. 1962. Testicular tumours in the dog. Journal of Comparative Pathology and Therapeutics. 72: 247-265.

4 Gates O. 1937. Cutaneous metastases of malignant disease. American Journal of Cancer. 30: 718.

5 Grem J.L., Robins H.I., Wilson K.S., Gilchrist K. \& Trump D.L. 1986. Metastatic leydig cell tumor of the testis report of three cases and review of the literature. Cancer. 58(9): 2116-2119.

6 Hayes H.M., Wilson G.P., Pendergrass T.W. \& Cox V.S. 1985. Canine cryptorchism and subsequent testicular neoplasia: case-control study with epidemiologic update. Teratology. 32(1): 51-56.

7 Hayes H.M. \& Pendergrass T.W. 1976. Canine testicular tumors: epidemiologic features of 410 dogs. International Journal of Cancer. 18(4): 482-487.

8 Kim I., Young R.H. \& Scully R.E. 1985. Leydig cell tumors of the testis: a clinicalpathological analysis of 40 cases and review of the literature. The American Journal of Surgical Pathology. 9(3): 177-192.

9 Kennedy P.C., Cullen J.M., Edwards J.F., Goldschmidt M.H., Larsen S., Munson L. \& Nielsen S. 1998. Histological Classification of Tumors of the Genital System of Domestic Animals. Washington DC: Armed Forces Institute of Pathology, pp.15-24.

10 Laufer A. \& Sulman F.G. 1956. Estrogenic Leydig-cell tumor with multiple metastases in a dog; the problem of bisexual hormone production by gonadal cells. Journal of Clinical Endocrinology \& Metabolism. 16(9): 1151-1162.

11 Liao A.T., Chu P.Y., Yeh L.S., Lin C.T. \& Liu C.H. 2009. A 12-year retrospective study of canine testicular tumors. Journal of Veterinary Medical Science. 71(7): 919-923.

12 MacLanchlan N.J. \& Kennedy P.C. 2002. Tumors of the genital system. In: Meuten D.J. (Ed). Tumors in Domestic Animals. 4th edn. Ames: Iowa State Press, pp.547-574.

13 Owston M.A. \& Ramos-Vara J.A. 2007. Histologic and immunohistochemical characterization of a testicular mixed germ cell sex cord-stromal tumor and a leydig cell tumor in a dog. Veterinary Pathology. 44(6): 936-943.

14 Peters M.A., Teerds K.J., van der Gaag I., de Rooij D.G. \& van Sluijs F.J. 2001. Use of antibodies against LH receptor, $3 \beta$-hydroxysteroid dehydrogenase and vimentin to characterize different types of testicular tumour in dogs. Reproduction. 121(2): 287-296.

15 Radi Z.A. \& Miller D.L. 2005. Immunohistochemical expression of calretinin in canine testicular tumours and normal canine testicular tissue. Research in Veterinary Science. 79(2): 125-129.

16 Ro J.Y., Kim K., Shen S.S., Amin M.B. \& Ayala A.G. 2013. Tumors and tumors-like conditions of the male genital tract: testicular neoplasms. In: Fletcher C.D.M. (Ed). Diagnostic Histopathology of Tumors. 4th edn. Philadelphia: Elsevier, pp.951-1009.

17 Spugnini E.P., Bartolazzi A. \& Ruslander D. 2000. Seminoma with cutaneous metastases in a dog. Journal of the American Animal Hospital Association. 36(3): 253-256.

18 Takiguchi M., Iida T., Kudo T. \& Hashimoto A.J. 2001. Malignant seminoma with systemic metastases in a dog. Small Animal Practice. 42(7): 360-362.

19 Togni A., Rütten M., Bley C.R. \& Hurter K. 2015. Metastasized Leydig cell tumor in a dog. Schweizer Archiv fur Tierheilkunde. 157(2): 111-115. 